\title{
Distribution of tissue progenitors within the shield region of the zebrafish
}

\section{gastrula}

\author{
John Shih and Scott E. Fraser* \\ California Institute of Technology, Division of Biology, Beckman Institute, Mail Code 139-74, Pasadena California 91125, USA \\ *Author for correspondence
}

\section{SUMMARY}

The zebrafish has emerged as an important model system for the experimental analysis of vertebrate development because it is amenable to genetic analysis and because its optical clarity allows the movements and the differentiation of individual cells to be followed in vivo. In this paper, we have sought to characterize the spatial distribution of tissue progenitors within the outer cell layers of the embryonic shield region of the early gastrula. Single cells were labeled by iontophoretic injection of fluorescent dextrans. Subsequently, we documented their position with respect to the embryonic shield and their eventual fates. Our data show that progenitor cells of the neural, notochordal, somitic and endodermal lineages were all present within the embryonic shield region, and that these progenitors were arranged as intermingled populations. Moreover, close to the midline, there was evidence for significant biases in the distribution of neural and notochord progenitors between the layers, suggesting some degree of radial organization within the zebrafish embryonic shield region. The distributions of tissue progenitors in the zebrafish gastrula differ significantly from those in amphibians; this bears not only on interpretations of mutant phenotypes and in situ staining patterns, but also on our understanding of morphogenetic movements during gastrulation and of neural induction in the zebrafish.

Key words: gastrulation, fate map, spatial pattern, cell lineage, videomicroscopy

\section{INTRODUCTION}

Fate maps depict what cells in various regions of an embryo will become during normal development. Although they do not by themselves tell us whether cells are committed in either the dynamic or material sense (Slack, 1991; Spemann, 1938), they do provide insight into the mechanisms of cell fate determination, embryonic induction and tissue morphogenesis. Construction of a fate map requires a means of labeling a cell (or distinct group of cells) in a defined region of the embryo, identifying the progeny of the labeled cell(s) over time and scoring the final phenotypes and positions of the progeny. As not all stages of any embryo are equally accessible for either labeling or scoring, fate maps are usually less than perfect. Recently, several fate maps have been constructed for the zebrafish embryo. Those of early cleavage stages have raised questions concerning early patterning events in these embryos and whether such events could be correlated with cleavage planes (Strehlow et al., 1994; Strehlow and Gilbert, 1993; Helde et al., 1994; Abdelilah et al., 1994). For the gastrula, Kimmel's fate map has been particularly useful (Kimmel et al., 1990), because it has facilitated experiments in cell transplantation, aided in the interpretation of staining patterns and served as an important tool in the experimental analysis of mutant phenotypes.

Kimmel and his colleagues proposed two reasons that the onset of gastrulation (about 50\% epiboly; 5.25 hours) is the earliest stage for which a fate map of clear predictive value could be made for the zebrafish: First, cell fates were thought to become fate-restricted near this stage (Kimmel and Warga, 1986; Kimmel et al., 1990). Second, the appearance of the embryonic shield just before 6 hours of development at the dorsal midline provides a reliable landmark for judging the position of labeled cells. Even though this gastrula fate map is based on a relatively small number of cells, the overall pattern prompted them to propose that the zebrafish gastrula fate map is more similar to those of other chordates than previous teleostean fate maps (Kimmel et al., 1990). Interestingly, the data in Kimmel's gastrula fate map suggest that the distributions of axial mesodermal and neural progenitors overlap. This apparent overlap could arise from the fact that single cells were labeled during blastula stages; thus, each point on their gastrula map represented small clones of cells instead of single cells. If such clones included progenitors for different cell types, an apparent progenitor overlap would result. Furthermore, the overlap might result from alignment errors or statistical limitations. It is important to resolve whether and to what extent progenitor populations overlap in the zebrafish gastrula fate map. Even limited intermixing between axial mesodermal and neural precursors would permit different classes of cell-to-cell interactions between neural progenitors and their inducers.

In this paper, we have sought to better characterize the distribution of tissue progenitors within the embryonic shield region by labeling single cells at the onset of gastrulation. 
Comparative and statistical analyses of the observed tissueprogenitor distributions confirmed many expected general patterns in their arrangement, such as the notochord primordium centered on the dorsal midline. Surprisingly, progenitors of the endoderm, neurectoderm, notochord and somitic lineages were all present and intermingled within the shield region, even within five cell-diameters of the blastoderm margin. The topographical relationship of progenitors in the zebrafish embryo bears some general resemblance to the fate maps of other vertebrates; however, some aspects of progenitor distribution, such as the intermingling of progenitors, are clearly distinct from amphibians at a comparable stage.

\section{MATERIALS AND METHODS}

\section{Terminology}

\section{Epiblast and hypoblast}

In the literature, the terms 'epiblast', 'mesoblast' and 'hypoblast/ entoblast' are often used interchangeably with 'ectoderm', 'mesoderm' and 'endoderm' when describing germ layers (Nelsen, 1953). However, 'epiblast' and 'hypoblast' are also used to describe the bilaminar blastoderm stage in the early gastrula of many organisms. In this context, the meaning of 'epiblast' and 'hypoblast' are largely independent of germ layers and define relative position only; the 'epiblast' is the upper/outer layer and the 'hypoblast', the lower/inner layer (Shoenwolf, 1991; Nelsen, 1953). Although their use is commonplace in describing the bilaminar blastoderm stage, the prospective fate compositions of this 'epiblast' and 'hypoblast' are not consistent from one organism to the next (see Nelsen, 1953) and questions concerning the origin of the 'hypoblast' remain controversial. Moreover, it is not known if the visible interface between the two layers is stable in the sense that cells do not move between the layers except at the blastoderm margin and, there, only from the 'epiblast' into the 'hypoblast'. Moreover, the thickness of the 'epiblast' or the 'hypoblast' in cell number remains uncertain, as does the variability of their thickness within the blastoderm (or within the region of the embryonic shield) at any given stage. Given these caveats, we have decided to use the more historically correct and less restrictive 'primary-ectoderm' (Dettlaff, 1993) and 'primitive-hypoblast' (Wilson, 1889) in place of the more familiar 'epiblast' and 'hypoblast'.

\section{Shield and shield region}

In this study, we made use of the outline of the 'embryonic shield' to document the position of labeled cells at the onset of gastrulation. We draw an important distinction between the 'embryonic shield' and the 'shield region'. The embryonic shield is commonly defined in terms of the discernible outline of the dorsal primitive-hypoblast. Because there is no corresponding outline in the primary-ectoderm at the shield stage, it is not known where the boundaries of this 'prospective shield' are. Our use of the term 'shield' simply describes the outline of the dorsal primitive-hypoblast and the term 'shield region' describes an area including both primary-ectoderm and primitive-hypoblast surrounding the shield (Fig. 1).

\section{Embryo collection}

General maintenance, embryo collection and staging of the zebrafish (Danio rerio) were carried out according to the Zebrafish book (Westerfield, 1994). The embryos were kept at $28.5^{\circ} \mathrm{C}$ in $30 \%$ Danieau solution [full-strength Danieau is the same as amphibian Modified Niu-Twitty solution (Keller, 1991) with double the normal $\mathrm{CaCl}_{2}$ concentration], except during manipulation, injection and documentation, during which the temperature was no less than $23^{\circ} \mathrm{C}$.

\section{Defining the time and site of injection}

The zebrafish embryonic shield can be first identified at around 50\% epiboly along the margin of the blastoderm, as the germ-ring begins to take shape, 20-30 minutes before the shield stage (Westerfield, 1994; Fig. 1). We have defined this as the early-shield stage for the zebrafish. The early-shield stage is when the embryonic shield can first be clearly identified in profile view as a greater thickening of the blastoderm margin (first defined for Fundulus heteroclitus by Oppenheimer, 1936). The early-shield stage typically coincides both with $50 \%$ epiboly (5 hours and 40 minutes) and with the onset of germring formation.

\section{Cell labeling}

Single deep cells (DEL cells) were iontophoretically injected with fluorescent dextrans, beneath the enveloping layer (EVL) at the early-shield stage. 8-10 embryos were pipetted onto a bed of 3\% methyl cellulose (Sigma) in 30\% Danieau solution and gently oriented with a hairloop. Injections were done as previously described (Wetts et al., 1989) using omega-dot aluminosilicate electrodes with 100-200 M $\Omega$ resistance and a 4 namp positive current for up to 20 seconds. We used several different fluorescent dextrans (100 $\mathrm{mg} / \mathrm{ml}$ working concentration; Molecular Probes, Eugene, Oregon), including $10 \mathrm{kD}$ fluorescein lysine dextran (D-1820), $10 \mathrm{kD}$ rhodamine lysine dextran (D-1817) and their $3 \mathrm{kD}$ equivalents (D3306 and D-3308, respectively). Except that the smaller $3 \mathrm{kD}$ dextrans labeled the cells as brightly as the $10 \mathrm{kD}$ form in less than half the time, they were otherwise equivalent. We confirmed the labeling of single cells immediately after injection by epifluorescence microscopy. We included those injections where cells in the enveloping layer (EVL) were labeled together with a single DEL cell because the EVL no longer contributes to the embryo proper by 4 hours of development (Kimmel et al., 1990). The embryos were subsequently removed from the methyl-cellulose by several rinses with $30 \%$ Danieau solution. Injecting a dish of ten embryos required less than 20 minutes.

\section{Documentation}

The position of each labeled cell was documented in three views (Fig. 1) after the embryos were transferred to a Sylgard-based plate (Sylgard 182 silicone elastomer, Dow Corning, cured and then pretreated with $2 \%$ BSA solution) with precut triangular wells, filled with $30 \%$ Danieau solution. The entire process from injection to position documentation takes roughly a half hour; by this time, they were clearly shield stage. The positions and fates of labeled cells were documented using a SIT camera (Hamamatsu C2400) mounted on a Zeiss AxioPhot microscope. The images were averaged $(n=8$ frames) before storage on optical memory disk (Panasonic OMDR 3038) using the VidIm image-processing software (authored by Belford, Fraser and Stollberg). To normalize for slight variations in shield size, the distance from the dorsal midline to the lateral edge of the shield was subdivided into ten equal sections; we then subdivided this height into ten units. Thus, the $10^{\text {th }}$ position along the blastoderm margin represents the lateral edge of the shield and the $10^{\text {th }}$ position along the animal vegetal axis represents the height of the shield. Each position (defined by its marginal and height coordinates, $\mathrm{x} \bullet \mathrm{y}$; see Fig. 1) closely approximates an average cell diameter. In Figs 2 and 3, we assumed bilateral symmetry and reflected those injections made on the lefthand side of the midline $(n=22)$ over to the right.

Of 143 injections, we used 136 single-cell injections for the frontalview fate map and a partially overlapping 80 injections for depth analysis, based on the accuracy with which the $x \bullet y$ and depth of the injected cell were documented. Fewer injections were used for depth analysis because some labeled cells appeared at intermediate depths and slight tilting of the embryos at the time of documentation com- 
plicated depth determination in some cases. Those used in the depth analysis were evenly distributed over the shield region.

\section{Subdividing the map for analysis}

To evaluate progenitor distribution patterns, we subdivided our injections into three sets (animal, medial and lateral; see Fig. 1). The animal region was defined as those positions more animal than the fifth animalvegetal position. Positions vegetal to the animal region and lateral to the $7^{\text {th }}$ marginal position were defined as the lateral region; the remainder $(0-7 \cdot 0-5)$ was defined as the medial region. Because there are no known functionally significant anatomical boundaries within the shield region, this division is arbitrary. For depth analysis, we limited our analysis to the first three cell layers of the blastoderm, counting from the outermost layer of the DEL inwards, excluding the EVL. Since we injected the first cell that the micropipette encountered, as determined by the membrane potential recorded, it was very rare that we labeled cells deeper than the third layer (see Fig. 1: $\mathrm{z}$ dimension).

\section{Weighted analysis}

To permit a direct comparison of progenitors between regions, we calculated weighted frequencies, which take into account the number of times that each region was injected. The weighted frequency for a region was obtained by dividing the number of times a given progenitor type was labeled by the percentage of the total injections that were performed into that region.

\section{Statistical analysis}

We applied the $\mathrm{z}$ statistics test for the equality of two proportions
Fig. 1. Documentation and assignment of positions within the embryonic shield region. Each embryo was documented in three orientations: animal pole view (A); frontal view (B); profile view (C) (labeled cell shown in white in $\mathrm{A}, \mathrm{B}$ and $\mathrm{C}$, photographed as a single image using combined epifluorescence and transillumination). (A) In the animal pole view, the lateral edge of the shield (small pointer) can be identified by the transition in the thickness of the blastoderm, as can the approximate dorsal midline (large pointer). The distance, $x$, representing half of the shield's width (10-12 cell diameters), is subdivided into ten equal units (shown diagrammatically in $\mathrm{F}$ to normalize for slight size variations. (A) The labeled cell was seven positions from the midline. (B) In the frontal view of the same embryo, the distance (in cell diameters and crosschecked in profile view with respect to shield height for position) of the labeled cell from the blastoderm margin can be determined. (C) In side or profile view, the height of the embryonic shield $y$ is measured from the margin of the blastoderm (large pointer) to the animalmost point (smaller pointer) of the embryonic shield. Height y was also subdivided into ten equal units to normalize slight differences between embryos (schematized in D). In profile view (C), the depth $\mathrm{z}$ was determined by counting the number of average cell diameters the labeled cell was from the surface of the blastoderm. In C, the labeled cell was two positions from the margin and was in the third layer. $(\mathrm{D}, \mathrm{E})$ The term embryonic shield (s) describes the semicircular shaped thickening formed by the dorsal primitivehypoblast (ph; dark grey; D, frontal view; $\mathrm{E}$, profile view), and the shield region (sr) includes both the primitive-hypoblast and the primary-ectoderm (pe) just surrounding it (dark grey plus light grey regions in D and E). Thus, the position of any cell can be described by three spatial coordinates: an $\mathrm{x}$
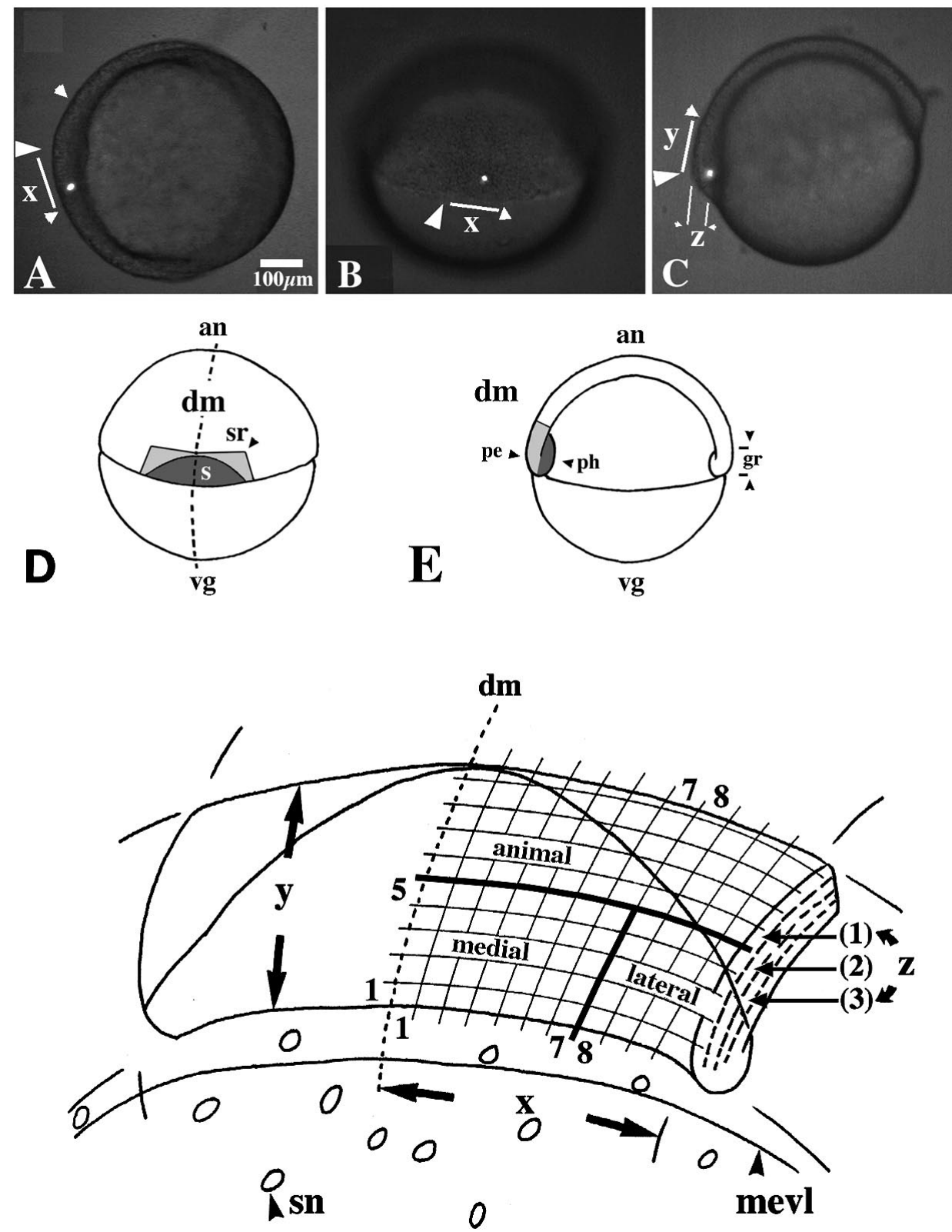

position along the margin, a y position along the animal-vegetal axis and a z position along the radial axis of the blastoderm, diagrammatically illustrated (F). Subregions used in the text for analysis are labeled animal, medial and lateral. mevl, margin of the enveloping layer; sn, yolk syncytial nuclei; dm, dorsal midline; an, animal pole; vg, vegetal pole; gr, germ ring. 
(Freund et al., 1960), which allows the comparison of two proportions (frequencies) derived from samples of a different size. We set the significance level at $P=0.05$. In the equation below, $X_{\mathrm{n}}=$ number of occurrences and $N_{\mathrm{n}}=$ sample size.

$$
\begin{gathered}
z=\frac{\frac{X_{1}}{N_{1}}-\frac{X_{2}}{N_{2}}}{\sqrt{\theta(1-\theta)\left[\frac{1}{N_{1}}+\frac{1}{N_{2}}\right]}} \\
\theta=\frac{X_{1}+X_{2}}{N_{1}+N_{2}}
\end{gathered}
$$

\section{Filming}

The bright-field time-lapse sequences were taken from longer recordings covering the duration of epiboly at $26^{\circ} \mathrm{C}$. Films were made on a Bio-Rad 500 laser scanning unit attached to a Zeiss Universal microscope using a $40 \times$ water-immersion objective. The embryos were seated in a triangular well cut into a $2 \%$ agar bed made up in $30 \%$ Danieau solution. They were gently rotated on their sides using an hairloop so that dorsal was upper most. They were each covered with a thin sheet of $1 \%$ agar $(\sim 50-100 \mu \mathrm{m}$ thick $)$, pinned down with cactus spines. Embryos held in this manner retained their rounded shape (coverslips can compress embryos, which alters normal cell movements), and could be held steadily in place for up to 10 hours with only occasional and limited rotations. Such embryos undergo and complete epiboly at the same rate as controls. Recordings were made at 1 minute intervals using a Kalman average of 4 frames, slow scan, and then stored on a optical memory disk recorder.

\section{RESULTS}

\section{Fates of the labeled progenitors}

After iontophoretic injection with fluorescent dextrans at the early-shield stage, the position of each single cell was documented in several orientations (Fig. 1). The labeled progeny that resulted were scored with respect to four different tissue types at multiple time points of development (Fig. 2). As expected, notochord progenitors were present within the embryonic shield region (Fig. 2A); the positions of cells that gave rise to notochord progeny are shown in Fig. 2A3. Not surprisingly, somitic progenitors were also present within the embryonic shield region (Fig. 2B). Interestingly, most of the labeled somite cells from our injections appeared to be either muscle pioneers or their immediate neighbors (positioned at the septum; Hatta et al., 1991; Hanneman and Westerfield, 1989; Patel et al., 1989). Neural progenitors also were found within the embryonic shield region (Fig. 2C). Labeled cells were typically found in the more ventral portions of the neural tube. Some of these cells differentiated to form the floorplate at the ventral mid-line. In addition, progenitors of the endodermal lineage were present within the embryonic shield region (Fig. 2D). The positions of those labeled progenitors that gave rise to endodermal progeny are shown in Fig. 2D3. We have no examples of hatching gland progenitors in our injections, although hatching gland progenitors are thought to overlap extensively with notochord progenitors (Kimmel et al., 1990). We believe they are absent from our sampling because hatching progenitors are already deep to the third cell layer by the early-shield stage putting them outside the scope of our distribution map. Our data set included only one case of head mesoderm (Fig. 2D3, position $3 \cdot 2$ counted as endoderm).

A comparison of the labeling frequency of various progenitors (Table 1) reveals that many of our injections labeled neurectodermal progenitors $(47 \%)$, a rate close to five times that of labeling endodermal progenitors $(10 \%)$ and nearly twice that of notochord precursors $(27 \%)$. In the shield region, there were nearly twice as many notochord progenitors as somite progenitors. Our attempt to correlate the rostrocaudal position of labeled progeny in the 19-20 hour embryo with progenitor position at the onset of gastrulation did not produce clear results. Using the somite positions as reference for scoring the progeny, we grouped the results into four classes: (1) rostral to somite one, (2) between the first and tenth somites, (3) between the tenth and twentieth somites, and (4) caudal to the twentieth somite. We found no obvious rostral-caudal order for any of the tissue types that we examined.

A comparison of the positions of the injections and the eventual fates of the cells suggests that each progenitor has a single fate, but that each position is not restricted to a single fate. In all cases, single cells labeled by iontophoretic injection at the early-shield stage gave rise to descendants of single tissue types. For example, positions $2 \bullet 3,3 \bullet 9$, and $10 \bullet 6$ (marginal position-animal-vegetal position) were each labeled only once, and each gave rise to neural descendants only (Fig. 2C4, 3B). Likewise, positions $4 \bullet 1,5 \cdot 3$ and $7 \bullet 1$ were also each labeled only once, and each gave rise to notochord progeny only (Figs 3B, 2A3). Table 2 shows that all of the 33 positions that were injected only once gave rise to clones of a single tissue type. Repeated injections in the same position tended to give rise to two or more different fates (Fig. 3A; Table 2). For example, of the two injections made into position $1 \bullet 8$, one gave rise to notochord progeny exclusively and the other gave rise to neural progeny exclusively (Figs 2A3, 2C4). A minority (5 of 13, Table 2 ) of the positions labeled by two different injections produced just one tissue type. For example, positions $1 \bullet 1011 \cdot 5$ and $17 \bullet 3$ each gave rise to neural progeny only (Fig.2C4). As expected, of the positions that were labeled by at least four different injections, most (6 of 10, Table 2) produced three

Table 1. Frequency of labeling a particular class of progenitors

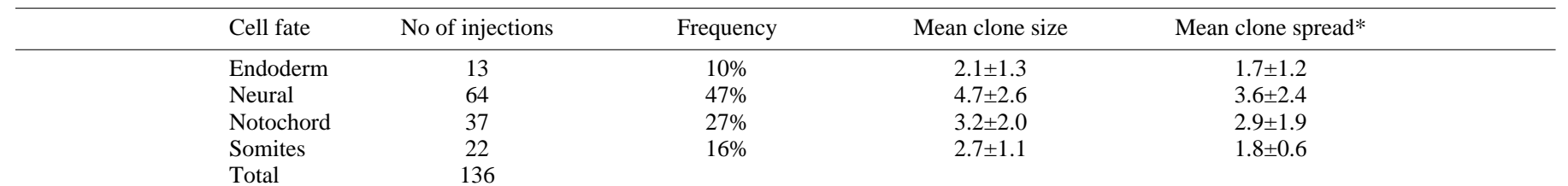

* Mean clone spread is presented in somite length units (length of a 4th or 5th somite) and is determined for 19-20 hour old embryo. 
Table 2. Correlation of number of injections per position in frontal view with number of fates

\begin{tabular}{lccccc}
\hline $\begin{array}{l}\text { No. of } \\
\text { injections }\end{array}$ & Total & 1 fate & 2 fates & 3 fates & 4 fates \\
\hline 1 & 33 & 33 & & & \\
2 & 13 & 5 & 8 & & \\
3 & 9 & & 7 & 2 & \\
4 & 4 & & 1 & 3 & \\
5 & 2 & & & 3 & 1 \\
6 & 4 & & & 3 & 1 \\
\hline
\end{tabular}

different fates, 3 of 10 produced all four possible fates (positions $8 \cdot 2,9 \cdot 3,11 \bullet 1$ and $13 \cdot 2$ ), and only one gave rise to two fates (position $2 \bullet 10$ ).

\section{Animal, medial and lateral sub-region comparisons}

To gain a better understanding of how the various progenitors were distributed within the embryonic shield region, we compared the labeling frequencies for the tissue progenitors between three subregions (animal, medial and lateral). The comparison revealed the following. First, progenitors of the notochord and neural lineages were present in all three regions (Table 3). Second, more than $30 \%$ of the neural progenitors in the medial and lateral regions gave rise to floorplate cells (see Fig. 2C4), while only $14 \%$ of the neural progenitors in the animal region did so. This observation suggests that there were more floorplate progenitors close to the blastoderm margin. Third, the weighted distribution of the progenitors (Table 4) reveals clear biases in the progenitor distribution. For example, more neural progenitors were found in the animal region than in the medial region. Analysis of both notochord and somitic progenitors shows that their labeling frequency values differed significantly between the medial and lateral regions, but not between the animal and medial regions (Table 3).

\section{Depth organization within the embryonic shield region}

The distribution of tissue progenitors within the depth of the zebrafish embryonic shield region was examined for the outermost three layers by correlating the positions of labeled cells within the thickness of the blastoderm (as seen in profile view) with their eventual fates (Table 5). In the lateral region, the labeling frequencies for the different progenitors were close to the same in each of the three layers. However, the distribution patterns in the medial region were quite different. Neural progenitors show a significant bias for the third layer from the

Table 3. Distribution of progenitors in frontal view

\begin{tabular}{lccc}
\hline Cell fate & Animal & Medial & Lateral \\
\hline Endoderm & $1(5 \%)$ & $5(16 \%)$ & $7(8 \%)$ \\
Neurectoderm* & $15(71 \%)$ & $12(38 \%)$ & $37(45 \%)$ \\
Notochord $\dagger$ & $5(24 \%)$ & $13(41 \%)$ & $19(23 \%)$ \\
Somitic meso $\dagger$ & $0(0 \%)$ & $2(6 \%)$ & $20(24 \%)$ \\
Total & $21(100 \%)$ & $32(100 \%)$ & $83(100 \%)$
\end{tabular}

Significance level $P<0.05$.

*Animal significantly different from medial.

$\dagger$ Medial significantly different from lateral.
Table 4. Weighted distribution frequency of progenitor between the three domains

\begin{tabular}{lccc}
\hline Cell fate & Animal & Medial & Lateral \\
\hline Endoderm & $17 \%$ & $54 \%$ & $29 \%$ \\
Neurectoderm* & $42 \%$ & $26 \%$ & $32 \%$ \\
Notochord $\dagger$ & $27 \%$ & $47 \%$ & $26 \%$ \\
Somitic Meso. $\dagger$ & $0 \%$ & $20 \%$ & $80 \%$ \\
& & \\
Significance level $P<0.05$. & \\
*Animal significantly different from medial. & \\
& \\
\hline
\end{tabular}

surface and notochord progenitors appeared to be concentrated in the second layer. These findings suggest some degree of radial organization (layering) within the medial portion of the embryonic shield region.

\section{Possible cause of the observed overlap}

Because the progenitor overlap observed in fate maps could be attributed in part to error in documenting the exact position of the labeled cells (Kimmel et al., 1990), it is necessary to address the role of alignment error in our study. Such variations may blur any boundaries in the map by a few cell diameters, but cannot fully account for the intermingling of neural and notochordal progenitors. First, there are significant numbers of neural progenitors in the medial subregion (Table 3); these cells were all well within the outline of the shield. Furthermore, of the 22 injections labeling cells at the margin, 9 labeled neural progenitors (41\%) and 8 labeled notochord progenitors (36\%). As it would be impossible to misidentify the blastoderm margin, the overlap there between tissue progenitors could not result from alignment difficulties.

Cell movement in the time surrounding our injections could contribute to the apparent overlap. During gastrulation, cells converge toward the dorsal midline as epibolic movements spread the blastoderm vegetalward over the surface of the yolk (Trinkaus et al., 1992; Kane and Warga, 1994; Warga and Kimmel, 1990). Could the concurrent veg-

Table 5. Number of injections made into each layer in the medial and lateral regions

\begin{tabular}{lccccccc}
\hline & \multicolumn{3}{c}{ Medial region $n=35$} & & \multicolumn{3}{c}{ Lateral region $n=45$} \\
\cline { 2 - 4 } \cline { 7 - 8 } Layers & 1 & 2 & 3 & & 1 & 2 & 3 \\
\hline Endo. & 4 & 2 & 3 & & 2 & 2 & 0 \\
Neuro. & 3 & 1 & 4 & & 4 & 8 & 3 \\
Noto. & 3 & 9 & 3 & & 4 & 7 & 2 \\
Som. & 1 & 2 & 0 & & 4 & 5 & 4 \\
Total & 11 & 14 & 10 & & 14 & 22 & 9
\end{tabular}

\begin{tabular}{lcccccc} 
& \multicolumn{2}{c}{ Frequency of labeling per layer in the medial and the lateral regions } \\
Endo. & $36 \%$ & $14 \%$ & $30 \%$ & $14 \%$ & $9 \%$ & $0 \%$ \\
Neuro. $\dagger$ & $27 \%$ & $7 \%$ & $40 \%$ & $29 \%$ & $36 \%$ & $33 \%$ \\
Noto. $* \dagger+$ & $27 \%$ & $64 \%$ & $30 \%$ & $29 \%$ & $32 \%$ & $22 \%$ \\
Som. & $9 \%$ & $14 \%$ & $0 \%$ & $29 \%$ & $23 \%$ & $44 \%$
\end{tabular}

In the medial region: significance level $P<0.05$.

*Layer 1 is significantly different from layer 2 .

$\dagger$ Layer 2 is significantly different from layer 3 .

†Layer 1 is significantly different from layer 3 .

The frequency values presented in this table are not weighted. 

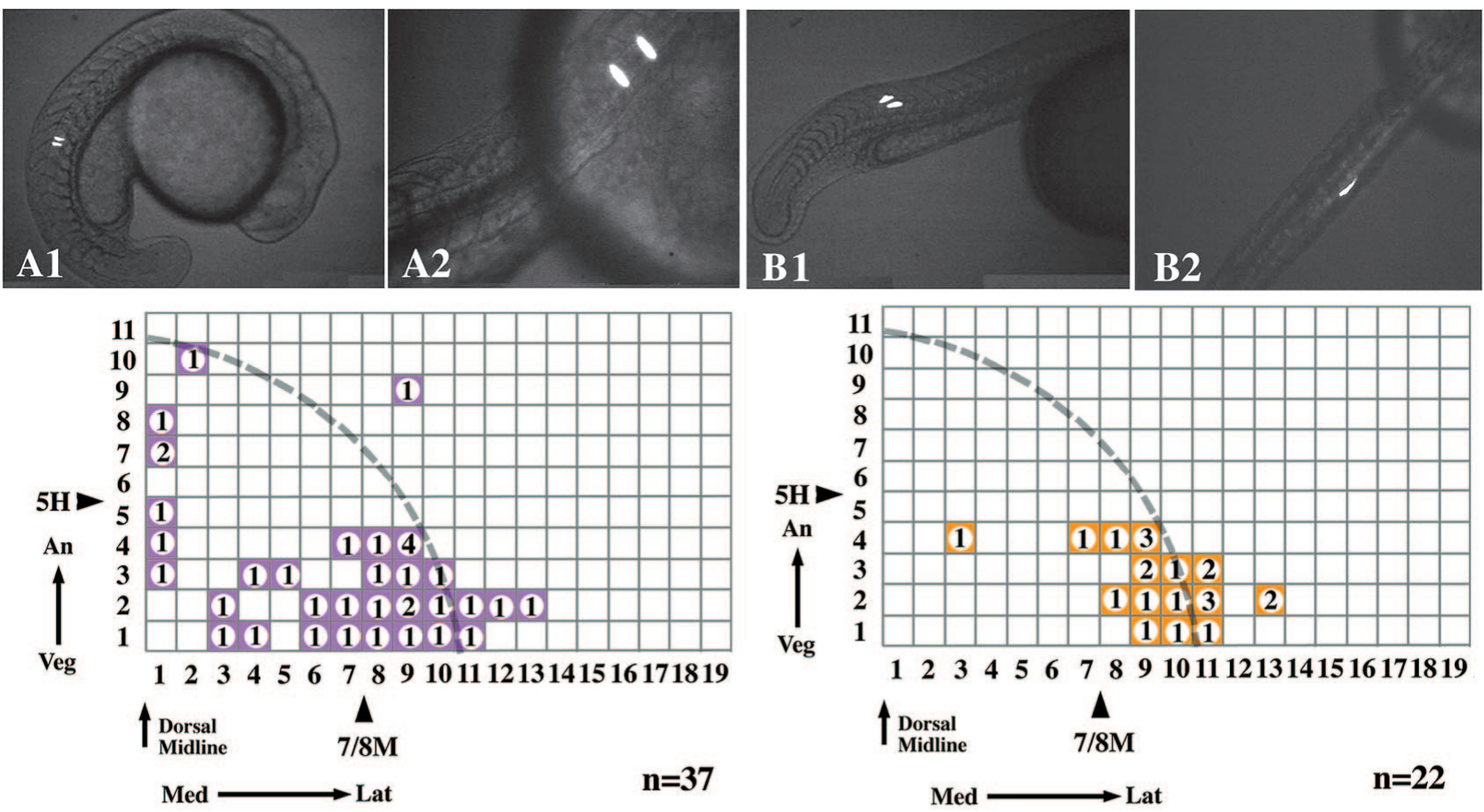

\section{A3}

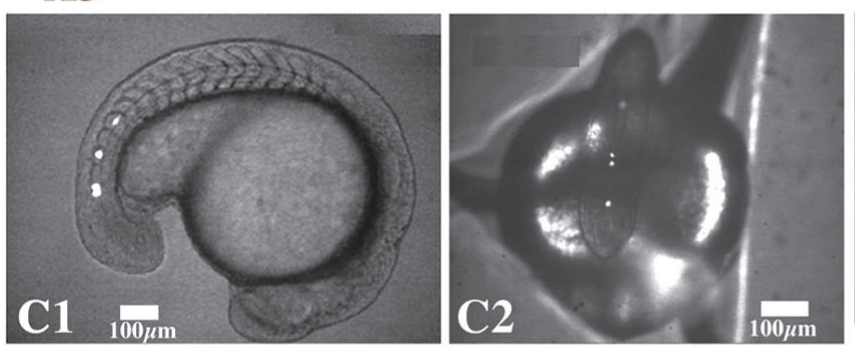

B3
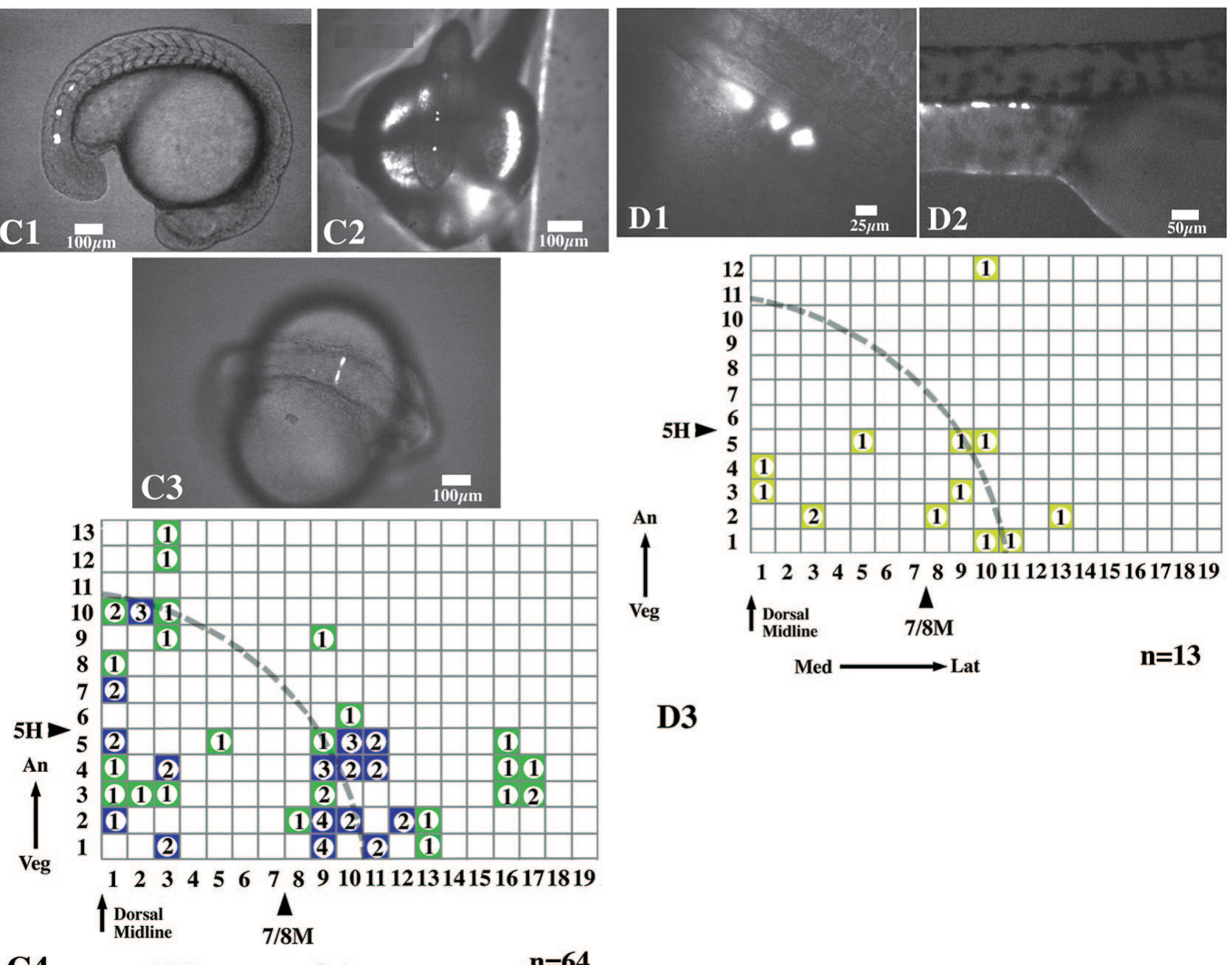

D3 
etalward and dorsalward movements of cells contribute to the presence of neural progenitors at the blastoderm margin? To address this possibility, we filmed one lateral half of the early-shield region ( $n=3$ recordings), the same region represented in the maps (Figs 1, 2 and 3). We could clearly resolve the advancing margin of the enveloping layer, the external yolk syncytial nuclei and outlines of all the cells within the first layer of DEL cells (Fig. 4A, C). We traced the movements of 57 cells over the half-hour period. While the margin of the enveloping layer (EVL) advanced about 2.5 cell diameters, the DEL-cell population advanced by less than 2 cell diameters vegetalward (Fig. 4). Cell neighbor relations were surprisingly well maintained. In Fig. 4, 49 of the 57 cells that we followed stayed on the surface during this time. The relative animal-to-vegetal positions of cells 4, 5, 9, 8, 57 and 49 at time 0 and 30 minutes were the same. The relative lateral-to-medial positions of cells 29, 13, 9, 8, 12, 40, 43 and 45 were the also maintained between the two time points. Relative movements of DEL cells and the yolk syncytial nuclei also appeared limited: the spatial relationships

Fig. 2. Assessment of cell fate and the distribution of progenitors. The fate of each labeled cell was determined by documentation at multiple time points (10 hours, 16 hours, $19-20$ hours and 24-30 hours). These four fates are: A, notochord; B, somitic mesoderm; C, neurectoderm; D, endoderm. Labeled cells (shown in white) were photographed as a single image using epifluorescence in combination with transillumination (not a photocomposite). After determining its fate(s), the position of a given progenitor was plotted onto a twodimensional grid representing the frontal view of one half of the embryonic shield centered on the dorsal midline (A3, B3, C4, D3). The numbers inside each square position represent the number of times that position was found to produce progeny of a given fate. Progenitors of the notochord lineage were identified by the disk-like morphology of the labeled progeny evident in side (A1) and top (A2) views. The positions of notochord progenitors were plotted on a grid in lavender (A3). Somite progeny appear as thin elongated cells oriented along the rostral-caudal axis in side (B1) or top (B2) view. Positions that produced somite progeny were labeled in orange (B3). The appearance of neural progeny was more varied (C1-3), but were consistently 'ventral' in position and character. Often we find a labeled contribution to the floorplate. Floorplate cells are located just above the notochord ( $\mathrm{C} 1$, the same embryo as in Fig. $1 \mathrm{C})$ and form a unicellular row when viewed from the top (C2). In contrast, other cells in the neural tube are not found at the midline (C3). In C3, the labeled cells lie bilaterally in the posterior rhombencephalon. Positions that produced neural progeny are shown in C4: blue indicates floorplate; green designates other neural derivatives. Only position $1 \bullet 2(\mathrm{x} \bullet \mathrm{y})$ gave rise to floorplate exclusively, and this position was injected only once. 15 of 39 positions contributed to both floorplate and other neural progeny. 23 of 39 positions gave rise to non-floorplate neural progeny. Endodermal progeny initially appear as flat cells that lie ventral-lateral to the notochord and ventral to somitic mesoderm (D1). Later on, they appear more rounded and are found along the interface between the mesoderm and the yolk (D2). In all but one instance (position 10•12), endodermal progenitors were located within five positions of the blastoderm margin. The positions where endodermal progenitors were labeled were marked in yellow (D3). $5 \mathrm{H}$ marks the location of the $5^{\text {th }}$ positions from the margin. $7 / 8 \mathrm{M}$ marks the location of the $7-8^{\text {th }}$ position along the blastoderm margin. The gray, dashed line in A3, B3, C4 and D3 represents the idealized outline of the right half of the 'embryonic shield'. The animal in Fig. 2A1 was injected in position $4 \bullet 1 ; 2 \mathrm{~A} 2$ in $1 \bullet 5 ; 2 \mathrm{~B} 1$ and $2 \mathrm{~B} 2$ are of the same animal in $10 \bullet 2 ; 2 \mathrm{C} 1$ in $3 \bullet 4 ; 2 \mathrm{C} 2$ in $1 \bullet 2 ; 2 \mathrm{C} 3$ in $1 \bullet 5 ; 2 \mathrm{D} 1$ in $8 \bullet 2$; and $2 \mathrm{D} 2$ in $3 \bullet 3$. between yolk syncytial nuclei ( $\mathrm{SN}$ )-d and cell 24, between $\mathrm{SN}$-e and cell 16 , and between $\mathrm{SN}-\mathrm{f}$ and cell 48 were stable between the time points.

This analysis of cell movements in the outermost layer of DEL cells showed that the presence of neural progenitors within this cell layer in the shield region cannot be attributed to random cell movements or to the vegetalward invasion of neural progenitors. Instead, as the population of DEL cells moved vegetally and medially, their relative movement with respect to one
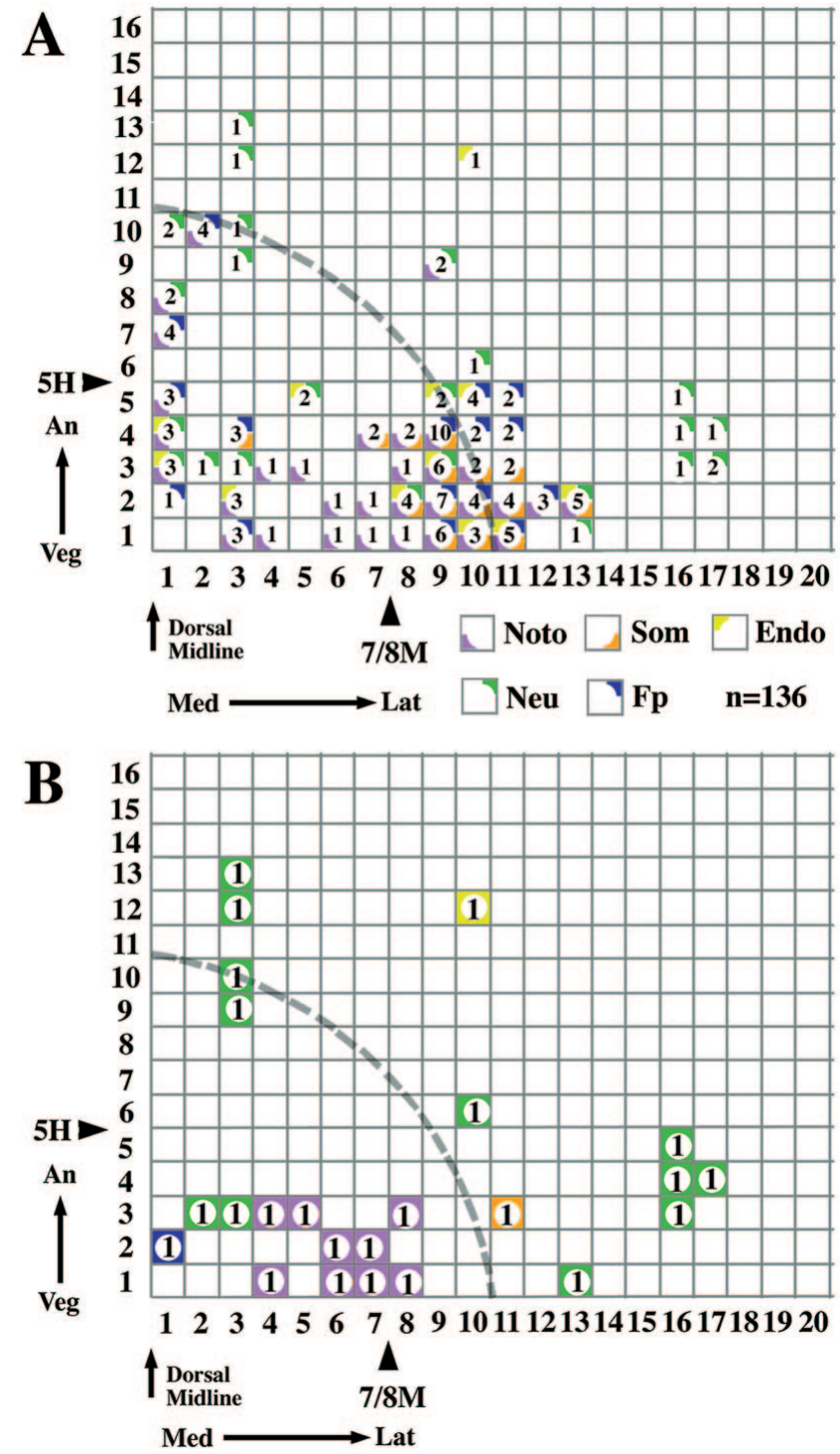

Fig. 3. Composite map of cell fate. (A,B) Summary composites of the progenitor distribution map in frontal view for all injections (A) and those positions injected only once (B). The horizontal and vertical axes are the same as those specified for the maps in Fig. 2; each position is represented as a square with five possible colors: yellow for endoderm progenitors, green for neural and blue for floorplate neural, lavender for notochord and orange for somites. The number at the center of each square indicates the number of times that position was labeled by injection. Only 24 of the singly-injected position are shown because reflecting the data points from the left hand side of the embryo over to the right resulted in nine single injections that overlapped. Noto, notochord; Som, somite; Endo, endoderm; Neu, neural: Fp, floor plate. 

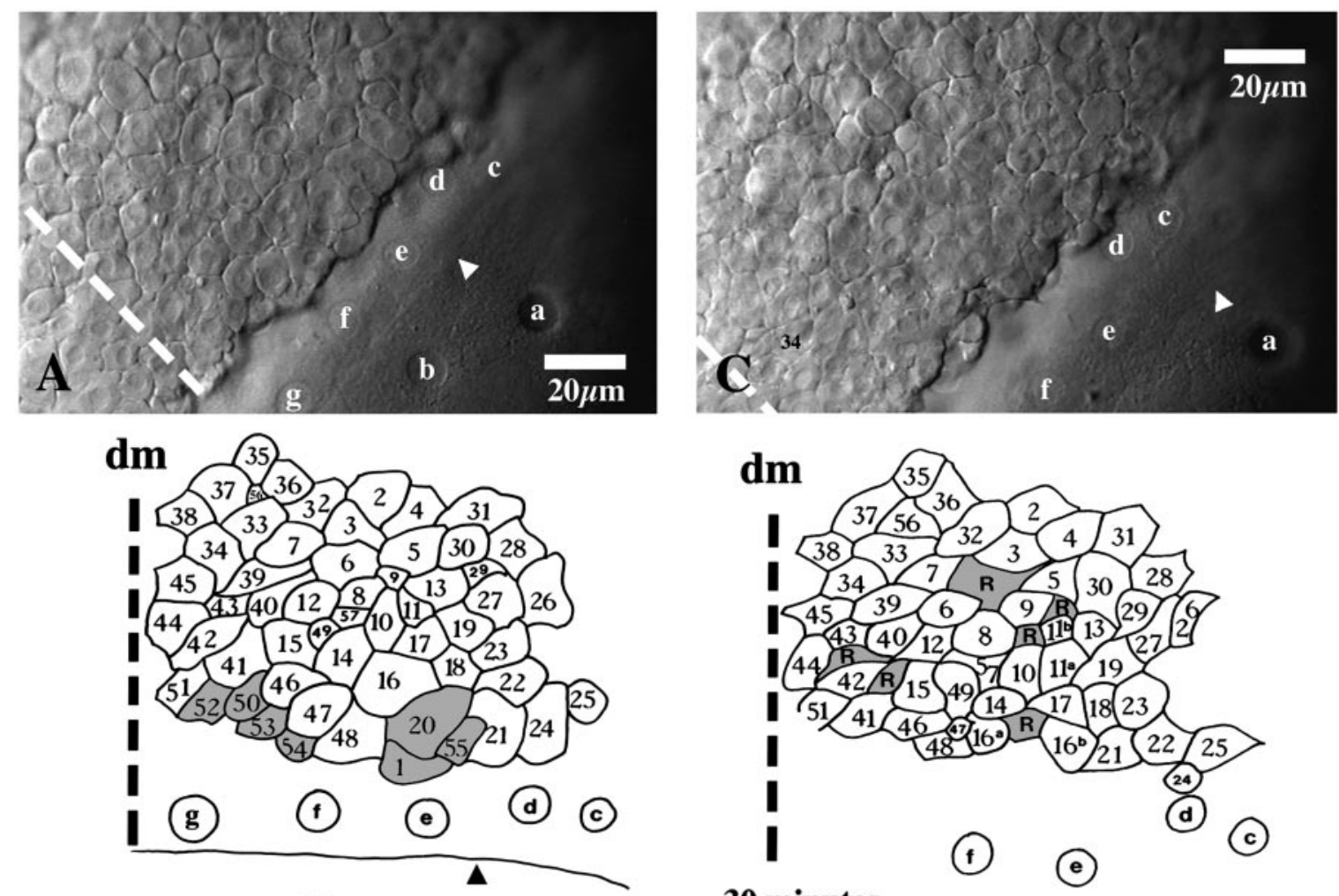

0 minutes
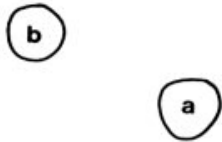

B

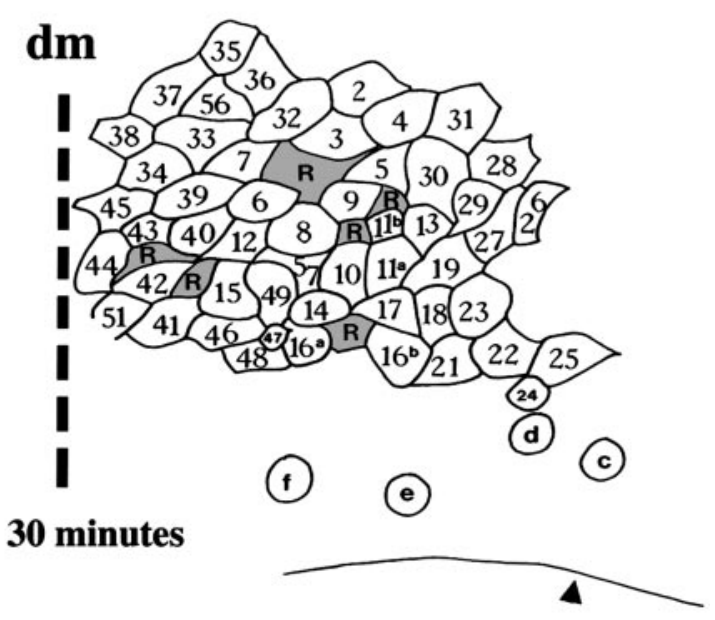

D

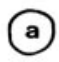

Fig. 4. Cell movement at early-shield and shield stage. We followed the movement of cells in the outermost DEL layer of half of the embryonic shield region between the early-shield stage ( 0 minute, A,B) and the shield stage (30 minutes, C,D). (A,C) The original screen image of the two time points; (B,D) tracings (here reoriented by $40^{\circ}$ clockwise; see dashed line representing the position of the dorsal midline). Of the 57 contiguous cells followed, the margin of the enveloping layer (pointer, white in A and C, black in B and D), and 8 syncytial nuclei (a-g). Six cells (shaded, numbered 1, 50,52,53,54 and 55) disappeared into deeper layers; 6/10 along the margin and one cell (shaded, numbered 20) located just behind the margin also moved out of the surface layer. Four of the ten marginal cells that we followed stayed at the surface during the half-hour period represented here. Six cells came into view from deeper layers during the same period of time (shaded, labeled R). The appearance of these cells was not clustered at the blastoderm margin but was distributed more randomly within the field of view. Of the 57 cells that we followed, 49 stayed on the surface. Two yolk syncytial nuclei moved off screen during the 30-minute period, suggesting a net movement towards the dorsal midline ( $\mathrm{dm}$, dashed line to the left, black in B and D, and white in A and C). The spatial relationship between yolk syncytial nuclei d (SN-d) and cell number 24; those between SN-e and cell number 16, and SN-f and cell number 48 stayed in close register between the time points.

another was minimal. If diagrams of the two time points are superimposed, using cell 51 and cell 25 as points of reference, almost all other cells overlap; therefore, the relative movement in this first layer of DEL cells is no more than one cell diameter during this time. Furthermore, greater than $90 \%$ of the total height (3.25/3.5 units, respectively) represented by the 57 cells we followed was retained during the half-hour period. While this analysis did not address whether deeper layers within the shield region moved in a similarly ordered fashion, it is reasonable to assume that the behavior of most cells within the blastoderm during this half-hour window should be very similar. Because the relative positions of the cells in the outermost DEL layer are nearly identical at the time of injection and position documentation, cell rearrangements cannot explain all of the observed overlap between tissue progenitors.

\section{DISCUSSION}

In this paper, we have sought to better characterize the distribution of tissue progenitors within the embryonic shield region at the onset of zebrafish gastrulation, concentrating on the outermost three DEL-cell layers of the shield region. As expected, single cells yield progeny that are tissue restricted. Most of the progenitor cells of the notochord were centered at the dorsal midline and were flanked laterally by somitic progenitors. However, contrary to expectations, progenitors of the endodermal, neural, notochordal and somitic lineages were intermingled within this region. There appears to be no absolute relationship between position and cell fate; instead, the number of different fates observed at a given position increases with the number of times that a position is sampled. 
The intermingling of progenitor populations cannot be dismissed as merely experimental error, or be explained simply by cell movements between the time of injection and the time of position documentation. Thus, the intermingling present in our data appears to reflect accurately the fate map of the shield region. Such intermingling within the shield region is significant as it would permit interactions of different timing and mechanism than typically discussed.

\section{Comparison of vertebrate gastrula fate maps}

It is commonly believed that the topological fate relationships in the vertebrate gastrulae are conserved (Kimmel et al., 1990; Lawson et al., 1991; Lawson and Pedersen, 1992) and that the zebrafish gastrula fate map more closely resembles those of other vertebrates than do fate maps of teleosts previously reported by others (see Kimmel et al., 1990). The rationale for the proposed topological conservation consists of three key features: First, the notochord anlage is centered at the dorsal midline. Second, there is somitic mesoderm on either side of the notochord primordium. Third, the neural primordium is located closer to the presumptive anterior end of the embryo than is the mesoderm. Although there have been reports of topological differences between teleostean fate maps (discussed in Collazo et al., 1994), these differences have been attributed to experimental limitations and are not thought to reflect true differences in tissue topology (Kimmel et al., 1990). The distribution of notochord and somitic progenitors that we report here is consistent with the first two key features. However, the intermingled relationship between mesoderm and neurectoderm progenitors in the zebrafish gastrula argues against a strict conservation of topological fate relationships in vertebrate embryos. Except for limited statistical overlap, intermingling of neural and mesodermal progenitors does not occur in amphibian fate maps (Vogt, 1929; Pasteels, 1940; Keller, 1975, 1976). Interestingly, data collected for the mouse (Lawson et al., 1991; Lawson and Pedersen, 1992) and chick (Selleck and Stern, 1991) fate maps show that a single labeled cell can give rise to both neural and mesodermal progeny, strongly suggesting that neural and mesodermal progenitors intermingle during gastrulation. Whether the zebrafish progenitor distribution map that we have presented is representative of a class of vertebrate fate maps that includes the chick and mouse, but which is distinct from those of the amphibians, remains to be determined.

The presence of neural progenitors in the shield region may alter some of the current interpretations of in situ hybridization or antibody-staining patterns in the zebrafish gastrula. For example, at 50\% epiboly, whole-mount antibody staining of the Brachyury gene product, Zf-T, labels cells described as endodermal and mesodermal progenitors, as well as cells of the enveloping layer within the blastoderm margin (SchulteMerker et al., 1992; their Fig. 6D,E represents early shield stage). Since all the nuclei within the first five-cell diameters of the blastoderm margin stain for $\mathrm{Zf}-\mathrm{T}$, it follows then that some neural progenitors must also express $Z f-T$ at one time. Interestingly, it appears that the $T$ gene is briefly expressed in neurectoderm progenitors during mouse and chick development (Kispert and Herrmann, 1994; Kispert et al., 1995). The absence of the Brachyury gene product in the neuronal domain of the frog fate map (Smith et al., 1991) highlights the impor- tance of comparative analyses in exploring the relationships between molecular events and cell fate.

\section{Patterns in the radial dimension}

There has been some debate regarding whether the teleostean gastrula fate map is organized radially, in layers along the thickness of the blastoderm. Radial organization (layering) of the teleostean blastoderm was proposed by Ballard to explain the overlaps in his fate map data of Salmo gairdneri embryos (Ballard, 1973). He proposed that the neural primordium was closest to the surface and the mesoderm and endoderm were in layers below (Ballard, 1973). Pooling data from injections made between 3.2 and 5.2 hours, Kimmel and colleagues pointed out that since DEL cells at different depths in the zebrafish blastula could contribute to varied and overlapping progeny, there was no evidence for radial organization (Kimmel et al., 1990). Furthermore, they reasoned that radial thinning during epiboly would be expected to stir up any depth organization within the blastoderm. Our injections, made into single cells of the embryonic shield region, reveal a subtle but significant radial organization. The clearest illustration of this layering is the distribution of neural progenitors, which shows a significant difference between the second and third cell layers.

It is not unusual for fate maps of vertebrate embryos to show radial organization. In amphibian embryos, it is common to find presumptive endoderm overlying presumptive mesoderm along the suprablastoporal margin (Nieuwkoop and Florcshütz, 1950; Keller, 1975, 1976; Pasteels, 1940; Vogt, 1929). Endoderm progenitors within the surface-most layer may be regarded, by analogy, as the zebrafish equivalent of the amphibian's suprablastoporal endoderm (see Keller, 1975, 1976). By this analogy, we would expect to find the notochord anlage in the second layer of the zebrafish embryonic shield region. In agreement with that prediction, the labeling frequency of notochord progenitors in the second layer of the medial region is almost twice that found in the first layer and three times that of the third layer. However, the significant number of neural progenitors in the third layer of the blastoderm in the medial region spoils the analogy, as there are no neural progenitors deep to the notochord anlage in amphibians (see Keller, 1976; Pasteels, 1940; Vogt, 1929; Purcell and Keller, 1993). Interestingly, the relative position of neurectodermal and endodermal primordia at the dorsal midline in the zebrafish is opposite that proposed by Ballard for Salmo (Ballard, 1973). It is unclear what the significance of this difference might be, but it might suggest that developmental patterns are not exactly recapitulated from one teleostean species to another; in the same way, Xenopus differs from other urodele and anuran amphibians in that Xenopus lacks significant surface mesoderm (Keller, 1975).

\section{Cell commitment in the shield}

Any fate map describes only what the labeled cells differentiated into during development and not their states of commitment at the time of labeling; therefore, it is unclear whether our distribution map describes an intermingling of differently committed cells or one of wholly uncommitted cells. This is an important point, because, if a number of these cells are committed, then the interactions underlying neural induction and mesoderm dorsalization in the zebrafish may take place 
earlier than is presently believed. Consistent with the notion of some degree of early commitment, cells within the embryonic shield region display a considerable degree of molecular specialization (Strahle et al., 1993; Thisse et al., 1994; Hammerschmidt and Nusslein-Volhard, 1993; Xu et al., 1994; Krauss et al., 1993; Schulte-Merker et al., 1992) and some may be functionally specialized to have organizer capacity (see review: Ho, 1992).

In recent experiments, Ho and his collaborators have tested cell commitment in the zebrafish gastrula by transplanting single cells from the germ-ring into the animal pole. Because most of the transplanted cells could differentiate to form ectodermal derivatives, these experiments have been taken to show that cells near or at the blastoderm margin in the zebrafish gastrula were uncommitted to mesodermal fates at the onset of gastrulation (Ho and Kimmel, 1993). However, these experiments did not take into consideration any possible dorsalventral difference in mesoderm commitment along the blastoderm margin (suggested by goosecoid staining; Stachel et al., 1993). As a result, the few instances in which the transplanted cell gave rise to mesenchyme may reflect regional differences in mesodermal cell-fate commitment by the fifth hour of development. Furthermore, in view of the progenitor intermingling that we have observed within the embryonic shield region, it is possible that many of the cells used in the grafting experiments that were thought to be mesoderm precursors were, instead, ectodermal progenitors. Were this the case, some of the reported conversions of cell fate from mesoderm to ectoderm may not have occurred.

\section{Involution and ingression}

The intermingling of progenitors reported here poses the question of how such populations form the separate and distinct germ layers characteristic of postgastrula embryos. The term some have employed to describe gastrulation movements is 'involution'. 'Involution' is defined as the "flowing of a sheet of cells over the edge of an in-pocketing where invagination has occurred" (Trinkaus, 1984, p11). Perhaps the best example of 'involution' is the orderly movements of axial mesoderm and endoderm cells in Xenopus gastrulation during which all cells in the immediate marginal region 'involute' (Keller, 1986; Shih and Keller, 1992, 1994). In contrast, during zebrafish gastrulation, mesoderm and endoderm progenitors in the germ ring must move from the primary-ectoderm into the primitive-hypoblast, while neighboring neural progenitors appear to remain behind in the primary-ectoderm. Such a sorting movement is quite distinct from involution. Moreover, the zebrafish blastoderm margin does not form by invagination as does the Xenopus blastoporal lip. 'Invagination' is "the inpocketing of an unbroken cell sheet, at a localized region", (Trinkaus, 1984, p11). Therefore, the term 'involution' does not accurately convey the dynamics of cell movements during zebrafish gastrulation. Perhaps 'ingression', where cells "sink inside as individuals, changing their relations to each other in the process" (Trinkaus 1984, p11), is the more-appropriate term.

\section{CONCLUSION}

The importance of knowing the spatial relationship between the neural primordium and its inducers in early development is underscored by the current debate over the timing and mechanism of neural induction and subsequent patterning in Xenopus (Zimmerman et al., 1993; Guthrie, 1991; Sharpe and Gurdon, 1990; Kessler and Melton, 1994; Doniach, 1992; Sive et al., 1989; Ruiz i Altaba, 1994; Keller et al., 1992). In relation to the discussion at hand, the intermingling of neural and notochord progenitors in the early zebrafish gastrula raises the possibility that neural induction and patterning may already be underway by this stage. Furthermore, the distinction between planar and vertical interactions in Xenopus neural induction and patterning may not be equally meaningful in the zebrafish embryonic shield region, because both classes of interactions could essentially be short range in all directions. The strong conservation of genetic elements, such as Brachyury, in early vertebrate development has underscored the similarity in the developmental mechanisms of a number of organisms. When these similarities are not exact, experiments designed to probe these sometimes subtle differences between organisms may provide insight into the basis for evolutionary diversification. By demonstrating how developmental mechanism(s) might differ between species against a background of clear and conspicuous similarities, we hope to exploit the ways in which a fish is not a frog to gain better insights into both the conserved and specialized roles of the many genes important to early development.

The authors thank Dr Bill Trevarrow for introducing us to the zebrafish as an experimental system and the Beckman Institute for supporting our research. We also thank Drs J. P. Trinkaus, Ray Keller and Eric Davidson for their encouragement and insights, and Drs J. M. Oppenheimer and W. W. Ballard for their support of our research. Moreover, we thank Dr Andrés Collazo and Katherine Woo for their reading of the manuscript and many valuable discussions. We express our deep gratitude to Woods Hole Marine Biology Laboratory where we attempted impossible experiments and entertained crazy ideas. Finally, we thank Dian De Sha for her help in editing the manuscript. This research was supported by NIH (HD26864) and NIH PostDoctoral Training Fellowship 5T32 HD07257.

\section{REFERENCES}

Abdelilah, S., Solnica-Krezel, L., Stainier, D. Y. R. and Driever W. (1994). Implications for dorsoventral axis determination from the zebrafish mutation janus. Nature 370, 468-471.

Ballard, W. W. (1973). A New fate Map for Salmo gairdneri. J. Exp. Zoology 21, 391-399.

Collazo, A., Bolker, J. A. and Keller, R. E. (1994). A Phylogenetic Perspective on Teleost Gastrulation. The American Naturalist 144, 133-152.

Dettlaff, T. A. (1993). Evolution of the historical and functional structure of the ectoderm, chordamesoderm, and their derivatives in Anamnia. Roux's Arch. Develop. Biology 203, 3-9.

Doniach, T. (1992). Planar induction of Anteroposterior Pattern in the Central Nervous System of Xenopus laevis. Science 257, 542-545.

Freund, J. E., Livermore, P. E. and Miller, I. (1960). Manual of Experimental Statistics. Englewood, N. J. : Prentice-Hall.

Guthrie, S. (1991). Horizontal and Vertical Pathways in Neural Induction. Trends in Neuroscience 14, 123-126.

Hammerschmidt, M. and Nusslein-Volhard, C. (1993). The expression of a zebrafish gene homologous to Drosophila snail suggests a conserved function in invertebrate and vertebrate gastrulation. Development 119, 1107 1118.

Hanneman, E. and Westerfield, M. (1989). Early expression of acetylcholinesterase activity in functionally distinct neurons of the zebrafish. Journal of Comparative Neurology 284, 350-361. 
Hatta, K., Bremiller, R., Westerfield, M. and Kimmel, C. B. (1991). Diversity of expression of engrailed-like antigens in zebrafish. Development 112, 821-832.

Helde, K. A., Wilson, E. T., Cretekos, C. J. and Grunwald, D. J. (1994) Contributions of Early Cells to the Fate Map of the Zebrafish Gastrula. Science 265, 517-520.

Ho, R. K. (1992). Axis formation in the embryo of the zebrafish, Brachydanio rerio. Seminars in Developmental Biology 3, 53-64.

Ho, R. K. and Kimmel, C. B. (1993). Commitment of cell fate in the early zebrafish embryo. Science 261, 109-11.

Kane, D. A. and Warga, R. M. (1994). Domains of Movement in the Zebrafish Gastrula. Seminars in Developmental Biology 5, 101-111.

Keller, R. E. (1975). Vital dye mapping of the gastrula and neurula of Xenopus laevis. I. Prospective areas and morphogenetic movements of the superficial layer. Develop. Biol. 42, 222-241.

Keller, R. E. (1976). Vital dye mapping of the gastrula and neurula of Xenopus laevis. II. Prospective areas and morphogenetic movements of the deep layers. Develop. Biol. 51, 118-137.

Keller, R. E. (1986). The cellular basis of amphibian gastrulation. In: Developmental Biology: A Comprehensive Synthesis. Vol. 2. The Cellular Basis of Morphogenesis vol. (ed. L. Browder), pp. 241-327. New York: Plenum Press.

Keller, R. E. (1991). Early embryonic Development in Xenopus laevis. In: Methods in Cell Biology vol. 36 (ed. B. K. Kay and H. B. Peng), pp. 62-113. San Diego: Academic Press Inc.

Keller, R. E., Shih, J., Sater, A. and Moreno, C. (1992). Planar induction of convergence and extension of the neural plate by the organizer of Xenopus. Developmental Dynamics 193, 218-234.

Kessler, D. S. and Melton, D. A. (1994). Vertebrate Embryonic Induction Mesoderm and Neural Patterning. Science 266, 596-604.

Kimmel, C. B. and Warga, R. M. (1986). Tissue specific cell lineages originate in the gastrula of the zebrafish. Science 231, 365-368.

Kimmel, C. B., Warga, R. M. and Schilling, T. F. (1990). Origin and organization of the zebrafish fate map. Development 108, 581-594.

Kispert, A. and Herrmann, B. G. (1994). Immunohistochemical Analysis of the Brachyury Protein in Wild-Type and Mutant Mouse Embryos. Developmental Biology 161, 179-193.

Kispert, A., Ortner, H., Cooke, J. and Herrmann, B. G. (1995). The Chick Brachyury Gene: Developmental Expression Pattern and Response to Axial Induction by Localized Activin. Developmental Biology 168, 406-415.

Krauss, S., Concordet, J.-P. and Ingham, P. W. (1993). A Functionally Conserved Homolog of the Drosophila Segment Polarity Gene $h h$ is Expressed in Tissues of Polarizing Activity in Zebrafish Embryos. Cell 75, 1431-1444.

Lawson, K. A., Meneses, J. J. and Pedersen, R. A. (1991). Clonal analysis of epiblast fate during germ layer formation in the mouse embryo. Development 113, 891-911

Lawson, K. A. and Pedersen, R. A. (1992). Clonal analysis of cell fate during gastrulation and early neurulation in the mouse. In: Postimplantation development in the mouse. vol. 165 (ed. Wiley and Chichester), pp. 3-26.

Nelsen, O. E. (1953). Comparative Embryology of the Vertebrates. New York: McGraw-Hill Book Inc.

Nieuwkoop, P. and Florcshütz, P. (1950). Quelques caractères spéciaux de la gastrulation et de la neurulation de l'oeuf de Xenopus laevis, Daud. et de quelques autres Anoures. Arch. Biol. (Liege). 61, 113-150.

Oppenheimer, J. M. (1936). Processes of Localization in Developing Fundulus. J. E. E. M. 73, 405-444.

Pasteels, J. (1940). Recherches sur la facteurs initiaux de la morphógénèse chez les Amphibiens Anoures. IV. Centrifugation axiale d'œuf fécondé et insegmenté. Arch. Biol. 51, 335-386.

Patel, N. H., Martin-Blanco, E., Coleman, K. G., Poole, S. J., Ellis, M. C., Kornberg, T. B. and Goodman, C. S. (1989). Expression of engrailed Proteins in Arthropods, Annelids, and Chordates. Cell 58, 955-968.

Purcell, S. M. and Keller, R. E. (1993). A different type of amphibian mesoderm morphogenesis as in seen in Ceratophrys ornata. Development 117, 307-317.

Ruiz i Altaba, A. (1994). Pattern Formation in the Vertebrate Neural Plate Trends in Neuroscience 17, 233-243.
Schulte-Merker, S., Ho, R. K., Herrmann, B. G. and Nüsslein-Volhard, C. (1992). The protein product of the zebrafish homologue of the mouse T gene is expressed in nuclei of the germ-ring and the notochord of the early embryo. Development 116, 1021-32.

Selleck, M. A. J. and Stern, C. D. (1991). Fate mapping and cell lineage analysis of Hensen's node in the chick embryo. Development 112, 615-626.

Sharpe, C. R. and Gurdon, J. B. (1990). The Induction of Anterior and Posterior Neural Genes in Xenopus laevis. Development 109, 765-774.

Shih, J. and Keller, R. E. (1992). Patterns of cell motility in the organizer and dorsal mesoderm of Xenopus laevis. Development 116, 915-930.

Shih, J. and Keller, R. E. (1994). Involution-a current view. Seminars in Developmental Biology 5, 85-90.

Shoenwolf, G. C. (1991). Cell Movements in the Epiblast During Gastrulation and Neurulation in Avian Embryos. In Gastrulation: Movements, Patterns, and Molecules vol. (eds. R. E. Keller, W. H. Clark, Jr. and F. Griffin), pp. 128. New York: Plenum Press.

Sive, H. L., Hattori, K. and Weintraub, H. (1989). Progressive Determination During Formation of the Anteroposterior Axis in Xenopus laevis. Cell 58, 171-180.

Slack, J. M. W. (1991). From Egg to Embryo: Regional Specification in Early Development. Second Edition ed. Cambridge: Cambridge University Press.

Smith, J. C., Price, B. M. J., Green, J. B. A., Weigel, D. and Herrmann, B. G. (1991). Expression of a Xenopus homolog of Brachyury (T) is an intermediate-early response to mesoderm induction. Cell 67, 79-87.

Spemann, H. (1938). Embryonic Development and Induction. New York: Yale University Press.

Stachel, S. E., Grunwald, D. J. and Myers, P. Z. (1993). Lithium perturbation and goosecoid expression identify a dorsal specification pathway in the pregastrula zebrafish. Development 117, 1261-74.

Strahle, U., Blader, P., Henrique, D. and Ingham, P. W. (1993). Axial, a zebrafish gene expressed along the developing body axis, shows altered expression in cyclops mutant embryos. Genes and Development 7, 14361446.

Strehlow, D. and Gilbert, W. (1993). A fate map for the first cleavages of the zebrafish. Nature 361, 451-453.

Strehlow, D., Heinrich, G. and Gilbert, W. (1994). The Fate of the Blastomeres of the 16-Cell Zebrafish Embryo. Development 120, 1791-1798

Thisse, C., Thisse, B., Halpern, M. E. and Postlethwait, J. H. (1994) goosecoid Expression in Neurectoderm and Mesendoderm is Disrupted in Zebrafish cyclops Gastrulas. Developmental Biology 164, 420-429.

Trinkaus, J. P. (1984). Cells into Organs: The Forces that Shape the Embryo. Second Edition. Englewood Cliffs, N. J. : Prentice-Hall Inc.

Trinkaus, J. P., Trinkaus, M. and Fink, R. D. (1992). On the Convergent Cell Movements of Gastrulation in Fundulus. Journal of Experimental Zoology 261, 40-61.

Vogt, W. (1929). Gestaltanalyse am Amphibienkein mit örtlicher Vitalfarbung II. Teil. Gastrulation und Mesodermbildung bei Urodelen und Anuren Wilhelm Roux Arch. EntwMech. Org. 120, 384-706.

Warga, R. M. and Kimmel, C. B. (1990). Cell movements during epiboly and gastrulation in zebrafish. Development 108, 569-580.

Westerfield, M. (1994). The Zebrafish Book: A Guide For the Laboratory Use of Zebrafish (Brachydanio rerio). 2. 1 ed. Eugene: University of Oregon Press.

Wetts, R., Serbedzija, G. and Fraser, S. E. (1989). Cell lineage analysis reveals multipotent precursors in the ciliary margin of the frog retina. Developmental Biology 136, 254-263.

Wilson, H. V. (1889). The embryology of the sea bass (Serranus atrarius). Bull. United States Fish Commission. 9, 209-277

Xu, Q., Holder, N., Patient, R. and Wilson, S. W. (1994). Spatially regulated expression of three receptor tyrosine kinase genes during gastrulation in the zebrafish. Development 120, 287-299.

Zimmerman, K., Shih, J., Bars, J., Collazo, A. and Anderson, D. J. (1993). XASH-3, a novel Xenopus achaete-scute homolog, provides an early marker of planar neural induction and position along the mediolateral axis of the neural plate. Development 119, 221-232. 